\title{
Fußball gegen Vorurteile
}

\author{
Eine randomisierte kontrollierte Studie
}

Es kommt mittlerweile eher selten vor, dass im Fachblatt Science eine Arbeit publiziert wird, die nur einen einzigen Autor bzw. eine einzige Autorin hat, und die es zudem auf das Cover des Fachblatts bringt. Die Politikwissenschaftlerin und Konfliktforscherin an der US-amerikanischen Stanford Universität, Salma Mousa, hat dieses Kunststück fertiggebracht. Dies ist umso bemerkenswerter, als in Science vor allem naturwissenschaftliche Arbeiten publiziert werden und sozialwissenschaftliche Studien die Ausnahme sind. Der Inhalt der Studie ist brisant: Der Effekt von gemeinsamem Fußballspielen auf Vorurteile und kooperatives Verhalten von Christen gegenüber Muslimen. Der Studienort ist nicht weniger brisant: Das kurdische Irak.

Vorurteile gegenüber anderen Menschen beruhen oft darauf, dass man die Menschen nicht kennt. Ein Beispiel: In denjenigen Bundesländern, die am wenigsten Flüchtlinge aufgenommen haben, gibt es die meisten Vorurteile (und Ängste) gegenüber Flüchtlingen. Vor mehr als 60 Jahren formulierte der Harvard-Psychologe Gordon Allport die praktisch-therapeutische Konsequenz aus dieser Beobachtung [1]: Vorurteile können dadurch abgebaut werden, dass die Menschen die Möglichkeit bekommen, kooperativ und auf Augenhöhe einander kennenzulernen. Neben gemeinsamen performativen Künsten wie Theaterspiel ${ }^{1}$, konzertantes Musizieren ${ }^{2}$ und Tanzen gehört Sport - insbesondere Mannschaftssport - zu den gesellschaftlich gewünsch-

1 Das Wiener Kindertheater, seit mehr als einem Vierteljahrhundert unter der Leitung von Sylvia Rotter, hat hierzu vielfältige positive Erfahrungen gesammelt.

2 Das Orchester aus israelischen und palästinensischen Musikern unter der Leitung von Daniel Barenboim (West-Eastern Divan Orchestra) dürfte das bekannteste Beispiel für die vielfach beschriebenen, positiven sozialen Erfahrungen sein, die durch Musik möglich werden. ten und daher auch geförderten und inszenierten Anlässen für solche Begegnungen. ${ }^{3}$

Aber funktioniert das auch? - Die empirische Basis der Vermutung von Allport ist bis heute eher schwach, weswegen die hier vorgestellte Studie von Bedeutung ist. Es handelt sich um ein Feldexperiment, mit dem Kausalität nachgewiesen werden kann. Es wurde im Irak durchgeführt, einem Land, dessen etwa 30 Millionen Einwohner zu 97\% muslimischen Glaubens sind; nur noch etwa 3\% der Bevölkerung sind Christen orientalischer Prägung, die unterschiedlichen Glaubensrichtungen angehören: Jesiden, chaldäische Katholiken, sowie Anhänger der assyrischen Kirche des Ostens, der Alten Kirche des Ostens, der armenisch-apostolischen Kirche, römisch-katholischen Kirche, syrisch-katholischen Kirche, syrisch-orthodoxen Kirche von Antiochien, der assyrisch-evangelischen Kirche und weitere kleinere Glaubensgemeinschaften. Vor 100 Jahren waren noch $25 \%$ der Iraker Christen. Vor allem in den letzten Jahren nahm jedoch ihre Zahl um fast 2 Millionen ab, von denen die meisten noch immer als Flüchtlinge im Inland oder Ausland keinen festen Aufenthaltsort haben. So wurden im Rahmen der Einnahme von Mossul am 10. Juni 2014 durch die Terrorgruppe Islamischer Staat von Syrien und Irak (ISIS) etwa 100000 Christen aus Mossul ins angrenzende Gebiet der Kurden (Region um die Stadt Erbil) vertrieben. Die Autorin beschreibt die Situation in dieser Region wie folgt: „Viele Christen glauben, dass ihre muslimischen Nachbarn an diesen Razzien beteiligt waren. Dieser Verdacht hat Christen davon abgehalten, in befreite Gebiete zurückzukehren, hat die Unterstützung für Milizen zur Selbstverteidigung angeheizt und die Wahrscheinlichkeit von Vergeltungsmorden und zukünftigen Konflikte erhöht. Zugleich sind muslimische Gemeinschaf-

3 Die Olympische Idee - weitaus älter als die Theorie Allports - ist hier zu nennen, wenn dies auch heute nach unsäglicher Politisierung vor 85 Jahren und überbordender Kommerzialisierung seit 30 Jahren kaum noch zu erkennen ist. ten aus benachbarten Dörfern in christliche Enklaven eingewandert, was die Christen im Irak dazu veranlasst hat, die Verwässerung ihrer Kultur und Identität zu befürchten. Die christlich-muslimischen Beziehungen im Nordirak sind daher nach wie vor von gegenseitigem Misstrauen und sozialer Abgrenzung geprägt“ [3].

Um experimentell herauszufinden, welche Effekte gemeinsames Fußballspielen auf das Verhalten und Denken von Christen gegenüber Muslimen hat, wurden 3 Fußball-Ligen neu geschaffen, in der jeweils 14 Mannschaften (von 10 oder 11 christlichen Spielern), die es in der Region schon gab, gegeneinander spielten. Zunächst trat in jeder der 3 Ligen jede Mannschaft zu 13 Spielen an (also jeder gegen jeden), dann spielten die Besten in 2 Spielen nach k.o.-System einen Sieger aus.

Auf kleinen Spielfeldern wurde je nach Größe des Feldes mit entweder 6 oder 7 Feldspielern auf jeder Seite gespielt, die häufig ausgewechselt wurden, sodass jeder Spieler etwa die gleiche Zeit auf dem Feld verbrachte. Ein Spiel dauerte 2-mal 30 Minuten und jede Mannschaft spielte mindestens 13 Spiele über einen Zeitraum von 8 Wochen. Bezieht man die Aufwärm- und Abkühlphasen vor und nach dem Spiel mit ein, hatten alle Spieler mindestens 26 Stunden Zeit auf dem Spielfeld mit anderen Spielern verbracht. Die Autorin begründet das Fußballspielen systematisch dadurch, dass eine Fußball-Liga die Bedingungen erfüllt, die man als relevant für den erfolgreichen Aufbau gegenseitigen Vertrauens und Abbau gegenseitiger Vorurteile zwischen verfeindeten Gruppen erachtet. „Mannschaftskameraden mussten zusammenarbeiten, um ihr gemeinsames Ziel zu erreichen, die Spieler unterlagen der ausgleichenden Wirkung des Mannschaftssports, und lokale christliche Führer und Organi- 
sationen unterstützten die Ligen “4 [3]. Die Autorin und ihre Mitarbeiter rekrutierten vorhandene Fußballmannschaften mit ausschließlich Christen als Spielern. Da in der Gegend um Erbil auch Muslime leben und diese ebenso gern und oft Fußball spielen wie die Christen, wurden insgesamt 63 muslimische Spieler, bei denen es sich ebenfalls um aus ihrer Heimat Vertriebene handelte, rekrutiert und der Hälfte der Mannschaften (Experimentalgruppe) zugeordnet. Der anderen Hälfte wurden jeweils 3 Christen zugeordnet, sodass alle vorbestehenden Mannschaften um 3 Spieler erweitert wurden.

Die gesamte Intervention bestand also aus 3 Liegen mit insgesamt 42 Mannschaften und 459 Spielern, die Christen waren. In der Experimentalbedingung spielten 3 Spieler muslimischen Glaubens mit, in der Kontrollbedingung 3 Spieler christlichen Glaubens. Auf die Gleichheit der Spielstärke der christlichen und muslimischen Spieler wurde ebenso geachtet wie auf eine Gesamtatmosphäre, die dem Ziel der Intervention, bei Christen Vertrauen gegenüber Muslimen aufzubauen, förderlich war. „Den jeweiligen Mannschaftskapitänen wurde mitgeteilt, dass eine lokale christliche Gemeindeorganisation mit einer in den Vereinigten Staaten ansässigen Universität zusammenarbeitet, um eine Fussball-Liga für Vertriebene anzubieten und ihre Erfahrungen zu untersuchen. Den Teilnehmern wurde gesagt, dass die Gemeinschaftsbildung eines der Ziele der Liga sei und dass daher jedem bereits existierenden Team 3 zusätzliche Spieler zugeteilt wurden, die christlichen Glaubens sein konnten oder nicht, um unterschiedliche Gruppen einzubeziehen “5 [3].

4 "Teammates had to cooperate to achieve their shared goal, players were subject to the equalizing effect of team sports, and local Christian leaders and organizations endorsed the leagues."

5 "Captains were told that a local Christian community organization was working with a United States-based university to offer a soccer league for displaced people and to research their experiences. Participants were told that community building was one of the leagues' aims and, as such, each team would be allocated an additional three players who may or may not be Christian in an effort to include diverse groups."
Spieler mit christlichem Glauben machten also die Mehrheit der Spieler aus, und nur um diese Spieler ging es in der Analyse der Daten (Änderungen der Einstellungen der muslimischen Spieler gegenüber Christen wurden dennoch „exploratorisch“ erfasst). Die Gesamtsituation war damit die, dass eine (christliche) regionale Minderheit in der Liga die Mehrheit ausmachte, was den Abbau von Vorurteilen begünstigte. „Diese ethnische Zusammensetzung verminderte wahrscheinlich die Bedrohung zwischen den Gruppen und erhielt zugleich die Stärke der Identität der jeweiligen Teams “6 [3]. Alle Spieler in der Liga waren Vertriebene, sodass es nicht geschehen konnte, dass christliche Spieler mit ISIS-Anhängern konfrontiert wurden.

Im anschließenden Text (und in einer längeren Diskussion weiterer ethischer Fragen im 43-seitigen Supplement der Arbeit) scheint durch, dass die gesamte Untersuchung in einem politischen Spannungs- und einem moralischen Mienenfeld stattfand: „Es ist wichtig, darauf hinzuweisen, dass Christen in vielen Teilen der arabischen Welt marginalisiert (oder sogar verfolgt) werden, wo Vorurteile in einigen Teilen der muslimischen Mehrheit das größere Problem darstellen. Der Zugang des Forschungsteams zur christlichen Gemeinschaft und die erwarteten Vorteile des Aufbaus eines sozialen Zusammenhalts zwischen vertriebenen Gemeinschaften haben jedoch dazu geführt, dass in dieser Studie der Schwerpunkt auf die Meinung bzw. Haltung der Christen gegenüber Muslimen gelegt wurde“7 [3]. Vor Beginn der Liga wurden alle Spieler der bereits existierenden Mannschaften nach ihren Einstellungen gegenüber Muslimen gefragt und das Ergebnis wurde herangezogen, um die zusätzlichen Spieler (jeweils 3 Christen oder Muslime)

6 “This ethnic composition likely lowered perceived intergroup threat while preserving strong team identities."

7 "It is important to note that Christians are marginalized (or persecuted outright) in many parts of the Arab world, where prejudice among some segments of the Muslim majority poses the larger challenge. The research team's access to the Christian community, however, and the anticipated benefits of building social cohesion between displaced communities motivated the focus on Christian regard toward Muslims in this study." auf die Mannschaften so zu verteilen, dass die Mannschaften der Experimental- und Kontrollbedingung im Hinblick auf das Ausmaß der Vorurteile gegenüber Muslimen maximal ähnlich waren. DiesVerteilung der Spieler geschah dann per Los, also randomisiert.

Zusätzlich zu den 3 experimentellen Ligen wurde noch eine Kontroll-Liga mit 9 Mannschaften geschaffen, in der nur christliche Spieler gegeneinander spielten. Die Idee dahinter war, dass in den anderen 3 Ligen selbst Mannschaften mit nur christlichen Spielern (also die Kontrollgruppe) 7-mal gegen Mannschaften mit jeweils 3 Muslimen spielten und daher auch Kontakt mit Muslimen hatten. Die Kontroll-Liga ohne jeglichen Kontakt mit Muslimen wurde für exploratorische Vergleiche herangezogen.

Untersuchungsgegenstand (abhängige Variablen) war nicht nur die per Fragebogen erfasste Einstellung gegenüber Muslimen, sondern auch tatsächliche Verhaltensweisen in der realen Welt. So erhielten die Spieler nach Beendigung der Liga die Möglichkeit zu einem gemeinsamen Abendessen, bei dem auch Muslime eingeladen waren („mixed dinner“). Sie erhielten zudem einen Gutschein für ein Essen im ca. 40 Autominuten entfernten Mossul; wurden gefragt, ob sie mit Muslimen trainieren würden; sollten einen „besten Spieler“ unter den muslimischen Spielern auswählen; konnten den Dollar, den sie für das Ausfüllen des ersten Fragebogens erhielten, entweder für eine christliche oder für eine nicht religiöse Nichtregierungsorganisation (NGO) spenden; und konnten sich für ein weiteres gemischtes Team (Christen und Muslime) für eine künftige Liga anmelden ( $\triangleright$ Tab. 1). Gemessen wurden jeweils die Unterschiede in den tatsächlichen Verhaltensweisen in Abhängigkeit davon, ob die Spieler in einer Mannschaft der Experimentalgruppe (3 zusätzliche Muslime) oder Kontrollgruppe (3 zusätzliche Christen) waren. Zudem wurden auch die Einstellungen gegenüber Muslimen abgefragt, d. h. es wurde gefragt,

- was ihnen wichtiger ist, die nationale Identität (Iraker zu sein) oder die ethnische bzw. religiöse Identität;

- ob sie einen Schiiten oder Sunniten als Nachbarn tolerieren würden und

- ob sie Araber bzw. Muslime für das Leid der Christen verantwortlich machen. 
Als Kontrollvariablen wurden die bei solchen Studien üblichen Variablen (Alter, Bildungsgrad, Familienstand und Einkommen) sowie zudem der Kirchenbesuch und möglicher Missbrauch durch ISIS erhoben. Dies wurde zuletzt gefragt, „um Bahnungseffekte zu verhindern“, wie im Supplement zu lesen ist (S. S1). Die gesamte Studie war damit angelegt, um den Nachweis zu führen, dass nach einem gewaltsamen Konflikt durch gemeinsamen sportlichen Kontakt von Mitgliedern der Konfliktparteien ein tolerantes Verhalten wiederaufgebaut werden kann. Hat das funktioniert? - Ja und Nein!

Betrachtet man das Verhalten der christlichen Spieler gegenüber den muslimischen Mitspielern auf dem Spielfeld, so zeigten sich signifikante Unterschiede zwischen Spielern in der Experimentalbedingung im Vergleich zur Kontrollbedingung: Mit 3 Muslimen für 2 Monate in einer Mannschaft gemeinsam Fußballspielen (die Experimentalbedingung) bewirkte, dass man eher bereit war,

- wieder mit Muslimen zu spielen und tatsächlich spielte (dies wurde 6 Monate nach der Liga erfragt),

- einen Muslimen zum „besten Spieler“ wählen und

- sich für ein gemischtes Team auch Christen und Muslimen für die nächste Liga anzumelden ( $\mathbf{A}$ bb. 1, links).

Ging es jedoch um das Verhalten gegenüber anderen Muslimen im Allgemeinen, so ergaben sich weder bei

- einer gemeinsamen Party in der Nachbarschaft mit Familie und Freunden,

- noch bei dem Essen mit Muslimen in Mossul und

- ebenfalls nicht bei der Spende Unterschiede zwischen den beiden Gruppen ( Abb. 1, rechts).

Auch die erfragten Einstellungen änderten sich insgesamt nur wenig: Unabhängig von der Experimental- oder Kontrollbedingung traute man Muslimen nicht bei Geschäften, wollte sie eher nicht als Nachbarn haben und gab ihnen die Schuld am Leid vieler Christen. Lediglich bei der Frage der höheren Bedeutung nationaler Identität oder ethnischer bzw. religiöser Identität hatte die Experimentalbedingung einen hochsignifikanten $(p<0,001)$ Effekt dahin-

- Tab. 1 Abhängige Variablen im Hinblick auf Verhalten und Einstellungen von Christen gegenüber Muslimen und Erhebungs- bzw. Messzeitpunkt (nach Daten aus [3])

Verhalten gegenüber muslimischen Mitspielern

Wann nach Beendigung der Liga

mit Muslimen trainieren

6 Mon

Muslim zum „besten Sportler“ wählen

2 Wo bis 5 Mon

sich für gemischtes Team anmelden

2 Wo bis 5 Mon

Verhalten gegenüber anderen Muslimen

\begin{tabular}{l|l} 
in Mosul Essen gehen & 1 Mon bis 4 Mon
\end{tabular}

Gemeinsame Party mit den Mitspielern und deren Frau bzw. 3 Wo bis 5 Mon

Freunden

\begin{tabular}{l|l|l}
$1 \$$ Spende für die Kirche oder eine neutrale NGO & 2 Wo bis 5 Mon
\end{tabular}

Einstellungen gegenüber Muslimen

Ethnische und religiöse Gruppen sind arbiträr, wichtiger ist, 2 Wo bis 5 Mon Iraker zu sein

Muslim als Nachbar

2 Wo bis 5 Mon

Muslime Schuld am Leid der Christen

2 Wo bis 5 Mon

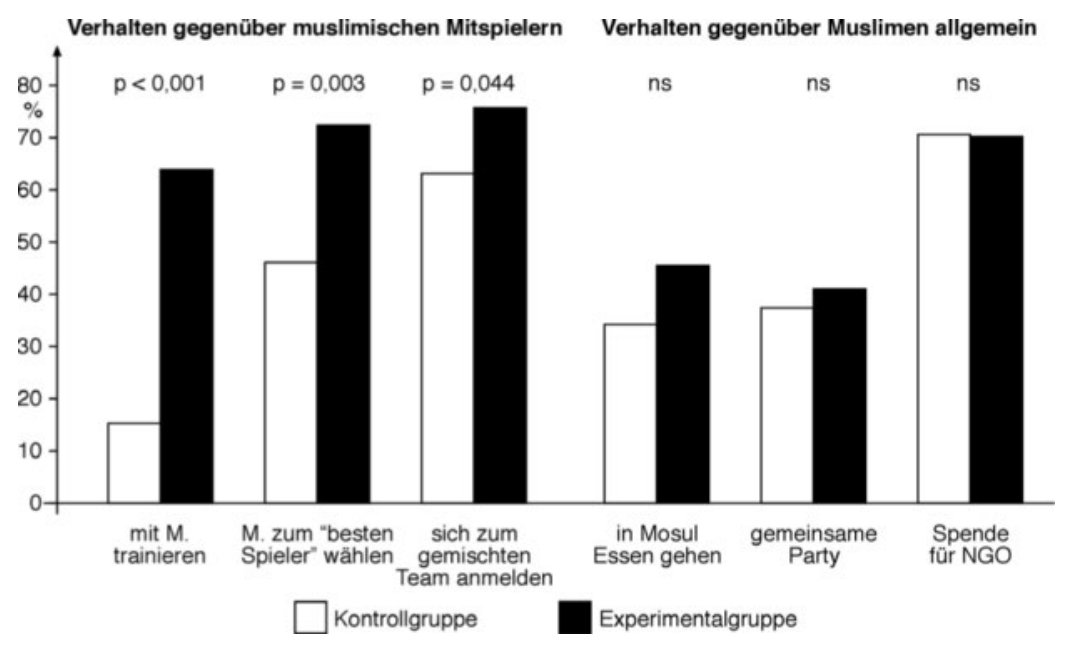

- Abb. 1 Ergebnisse auf der Verhaltensebene: Gegenüber muslimischen Mitspielern auf dem Fußballfeld kam es in der Experimentalgruppe zu (statistisch und sozial) bedeutsamen Änderungen im Verhalten (3 linke Säulen), nicht jedoch gegenüber Muslimen im Allgemeinen (nach Daten aus [3], Fig. 1, sowie den im Supplement, S. 29, tabellarisch angeführten Werten).

gehend, dass die Spieler in dieser Bedingung die religiöse bzw. ethnische Zugehörigkeit eher als arbiträr einstuften und die nationale Identität für bedeutsamer hielten. Exploratorische Analysen zeigten, dass

- in der Liga erfolgreiche Mannschaften signifikant stärkere positive Effekte aufwiesen, dass

- auch bei den Zuschauern der Liga positive Effekte zu verzeichnen waren und dass
- im Vergleich zu Vergleichsliga (ohne jegliche Beteiligung von Muslimen) die Effekte in den Ligen mit Experimentalund Kontrollbedingung größer waren. Dies bedeutet, dass Fußballspielen auch dann Vorurteile abbauen kann, wenn einige der von diesen Vorurteilen Betroffenen einfach nur mitspielen - und sei es in der gegnerischen Mannschaft.

Die exploratorische Analyse der während der Ligen verteilten gelben und roten Karten zeigte, dass es keine Unterschiede zwi- 
schen den Spielen von nur Christen untereinander und den Spielen von Christen gegen gemischte Teams gab. Dies ist bedeutsam, denn es zeigt, dass in den Ligen (und auf den Spielfeldern) die Probleme gegenseitiger Aggressivität ehemals verfeindeter Gruppen offenbar kein Thema waren. Es gab zudem keine Unterschiede in der Verteilung der Karten in Abhängigkeit von der Religionszugehörigkeit der Schiedsrichter. Schließlich zeigte sich bei den muslimischen Spielern keine Verstärkung von Vorurteilen gegenüber Christen. Umgekehrt war dies ebenso.

Zusammenfassend lässt sich feststellen, dass diese Studie durchaus positive Auswirkungen gemeinsamer sportlicher Aktivitäten finden konnte. Ihr experimenteller Charakter und die Tatsache, dass reales Verhalten registriert und ausgewertet wurde (und nicht nur Einstellungen erfragt wurden) machen die Ergebnisse valide, zeigen aber auch die Begrenztheit der Effektivität solcher vertrauensbildenden Maßnahmen. Vertrauen ist leicht verloren und nur schwer (und über lange Zeiträume) aufzubauen. Das liegt in seinem Wesen. Konkretes Verhalten im konkreten Miteinander ist leichter zu ändern als allgemeine Einstellungen gegenüber ehemals verfeindeten Gruppen. Wer miteinander Erfolg hat, ändert sein Verhalten und seine Einstellungen am ehesten. Offenbar brauchen Menschen Erfolge, um sich zu ändern. Diese Einsicht in die milliardenschweren Programmen zum friedlichen Miteinander zu implementieren, um deren Effektivität zu steigern (d. h. den Frieden kostengünstiger zu erreichen), stellt keine kleine Herausforderung dar.

Interessenkonflikt

Es liegen keine Interessenkonflikte vor.

\section{Korrespondenzadresse}

Prof. Dr. Dr. Manfred Spitzer

Universität Ulm

Abteilung für Psychiatrie

Leimgrubenweg 12-14

89075 Ulm

Deutschland
Literatur

[1] Allport G. The Nature of Prejudice: 25th Anniversary Edition. New York: Basic Books 1954/1979

[2] Dixon J, Levine M, Reicher S, et al. Beyond prejudice: are negative evaluations the problem and is getting us to like one another more the solution? Behav Brain Sci 2012; 35: $411-425$

[3] Mousa S. Building social cohesion between Christians and Muslims through soccer in post-ISIS Iraq. Science 2020; 369: 866-870

[4] Paluck EL, Clark CS. Can playing together help us live together? A field experiment in Iraq shows that having Muslim teammates reduced Christian soccer players' prejudice. Science 2020; 369: 769-770

\section{Bibliografie}

DOI https://doi.org/10.1055/a-1193-8288 Nervenheilkunde 2020; 39: 746-749 (C) 2020. Thieme. All rights reserved. Georg Thieme Verlag KG, Rüdigerstraße 14, 70469 Stuttgart, Germany ISSN 0722-1541 